\title{
Kikuchi-Fujimoto disease: lymphadenopathy in siblings
}

\author{
Allison Stasiuk BSc Pharm, Susan Teschke MD, Gaynor J. Williams MD MPhil, \\ Matthew D. Seftel MD MPH
}

\section{Patient 1}

A 19-year-old Aboriginal woman presented with a three-week history of swollen neck glands, nausea, vomiting, chills and weight loss. On examination, she had bilateral, nontender, diffuse cervical lymphadenopathy. Oral examination revealed extensive dental caries and periodontal disease. The results of her laboratory workup are shown in Table 1. Both a lymph node aspiration and a bone marrow biopsy were nondiagnostic. The patient was scheduled for a surgical lymph node biopsy, but when she was seen one month later, the lymphadenopathy had resolved spontaneously. Based on these findings, she was diagnosed with Kikuchi-Fujimoto disease. At follow-up a year and a half later, she had no evidence of recurrence.

\section{Patient 2}

Two years after the initial presentation of the first patient, her 19year-old younger sister was assessed for a three-week history of swollen neck glands, night sweats, decreased appetite and weight loss. The sister also had severe dental and periodontal disease. A computed tomography (CT) scan of the neck showed severe bilateral lymphadenopathy, with the largest lymph node measuring $2.6 \times 1.2 \times 2.6 \mathrm{~cm}$. Further CT scans showed moderate lymphadenopathy in the mediastinal, right hilar, axillary and pelvic regions. The results of a laboratory work-up are shown in Table 1. An excisional biopsy of a cervical lymph node and a bone marrow biopsy were done. By one month after presentation, the patient's symptoms and signs had resolved, and she was discharged home. She was also diagnosed with Kikuchi-Fujimoto disease. At follow-up seven months later, she had a normal physical examination and her laboratory test results were normal.

Given that both siblings presented with an uncommon disorder at the same age, human leukocyte antigen (HLA) genotyping was done to examine whether shared immunogenetics may account for a predisposition to this disease. The results are shown in Table 1.

\section{Pathology findings}

In both sisters, the results of bone marrow examination were normocellular for all lineages and showed no evidence of myelodysplasia or features of a neoplastic infiltrate. Aspiration

\section{Key points}

- Kikuchi-Fujimoto disease is an uncommon and sometimes familial disorder that should be considered in the differential diagnosis of cervical lymphadenopathy.

- Typical presentation includes fever, leukopenia and cervical lymphadenopathy.

- Although Kikuchi-Fujimoto disease is self-limiting and no definitive treatment exists, lymph node biopsy is required to rule out malignancy.

- Patients should be followed closely because of increased risk for recurrence and for systemic lupus erythematosus.

of a cervical lymph node in the first sister was nondiagnostic. In the second sister, an excisional biopsy showed geographic areas of necrosis containing apoptotic bodies and a striking degree of karyorrhexis with nuclear debris (Figure 1). Cells within the areas of necrosis were highly proliferative; more than $60 \%$ of the cells tested positive for the Ki-67 proliferation marker. Cellular material included a large number of histiocytes (CD68positive and myeloperoxidase-positive), some with crescentshaped nuclei, as well as large transformed lymphocytes (primarily CD8-positive T cells) (Figure 2). No neutrophils and only a few plasma cells were seen. Staining tests for organisms (periodic acid-Schiff reaction, Gömöri methenamine silver stain and Ziehl-Neelsen staining method) were negative. No morphologic evidence of malignancy was seen.

\section{Discussion}

Kikuchi-Fujimoto disease is also known as Kikuchi disease or histiocytic necrotizing lymphadenitis. It was first described in Japan by Kikuchi and Fujimoto in 1972, and has now been reported in most regions of the world. Typical presentation includes fever, cervical lymphadenopathy and leukopenia, but may also include night sweats, nausea, vomiting and weight loss. ${ }^{1}$ Lymphadenopathy is most commonly cervical (79\%), but can include any lymph node region and can be generalized

From the Faculty of Medicine (Stasiuk), the Department of Internal Medicine (Teschke, Seftel), the Department of Pathology (Williams), University of Manitoba, Winnipeg, Man.

CMAJ 2011. DOI:10.1503/cmaj.091347 
(Box 1). ${ }^{1}$ The median age at presentation is 30 years. Most patients $(70 \%)$ are young adults, but the disease can occur at almost any age. ${ }^{1,2}$ Reported case series support a female predilec-

Table 1: Results of laboratory investigations in two patients with Kikuchi-Fujimoto disease

\begin{tabular}{|c|c|c|}
\hline Investigation & Patient 1 & Patient 2 \\
\hline $\begin{array}{l}\text { Leukocyte count, } \times 10^{9} / \mathrm{L} \\
\text { (normal 4.5-11) }\end{array}$ & 2.8 & 1.9 \\
\hline $\begin{array}{l}\text { Lymphocyte count, } \times 10^{9} / \mathrm{L} \\
\text { (normal 1.0-3.3) }\end{array}$ & 0.88 & 0.50 \\
\hline $\begin{array}{l}\text { Neutrophil count, } \times 10^{9} / \mathrm{L} \\
\text { (normal 1.7-7.6) }\end{array}$ & 1.66 & 1.30 \\
\hline $\begin{array}{l}\text { Platelet count, } \times 10^{9} / \mathrm{L} \text { (normal } \\
1140-440 \times 10^{9} / \mathrm{L} \text { ) }\end{array}$ & 226 & 244 \\
\hline $\begin{array}{l}\text { Hemoglobin (normal 120-160 } \\
\text { g/L) }\end{array}$ & 109 & 107 \\
\hline $\begin{array}{l}\text { Mean cell volume (normal } \\
80-98 \mathrm{fL} \text { ) }\end{array}$ & 83.7 & 83.2 \\
\hline AST (normal 10-32 U/L) & 67 & 62 \\
\hline ALT (normal 0-25 U/L) & 29 & 18 \\
\hline ALP (normal 30-120 U/L) & 76 & 112 \\
\hline $\begin{array}{l}\text { Total bilirubin (normal 3-18 } \\
\mu \mathrm{mol} / \mathrm{L} \text { ) }\end{array}$ & 8 & 4 \\
\hline LDH (normal < 200 U/L) & 1128 & 1352 \\
\hline ESR (normal 0-30 mm/h) & 31 & Not performed \\
\hline CMV IgM & Negative & Negative \\
\hline CMV IgG & Positive & Not performed \\
\hline Hepatitis C serology & Negative & Negative \\
\hline Hepatitis B sAg & Negative & Negative \\
\hline HIV $1 / 2$ serology & Negative & Negative \\
\hline EBV IgM & Negative & Negative \\
\hline Bartonella henselae serology & Negative & Negative \\
\hline Francisella tularemia serology & Negative & Not performed \\
\hline Sputum stains for mycobacteria & Negative & Negative \\
\hline Anti-nuclear antibody & Negative & $\begin{array}{l}\text { Positive }(1: 160) ; \\
\text { repeat test } \\
\text { negative }\end{array}$ \\
\hline SSA (Ro) antibody & NA & Positive \\
\hline SSB (La) antibody & NA & Negative \\
\hline Sm antibody & NA & Positive \\
\hline RNP/Sm antibody & NA & Positive \\
\hline Scl-70 antibody & NA & Negative \\
\hline JO-1 antibody & NA & Negative \\
\hline dsDNA & NA & Negative \\
\hline HLA-A & 02 & 02,31 \\
\hline HLA-B & 39,35 & 39,51 \\
\hline HLA-CW & 7,4 & 07,15 \\
\hline HLA-DRB1 & 08,14 & 08,14 \\
\hline HLA-DQB1 & 03 & 03,0402 \\
\hline
\end{tabular}

Note: $A L P=$ alkaline phosphatase, $A L T=$ alanine transaminase,

$\mathrm{AST}=$ aspartate aminotransferase, $\mathrm{CMV}=$ cytomegalovirus, dsDNA = doublestranded deoxyribonucleic acid, EBV = Epstein-Barr virus, ESR = erythrocyte sedimentation rate, HLA = human leukocyte antigen, $\mathrm{LDH}=$ lactate dehydrogenase, RNP = ribonucleic protein, Scl = scleroderma, SS = Sjögren syndrome. tion with a male:female ratio ranging from $1: 4$ to $1: 1.3 .^{1,3}$ The disease is self-limiting, has no definitive treatment and spontaneously resolves within one to four months. The risk of recurrence is $3 \%-4 \%$ and can occur months to years after the initial diagnosis. ${ }^{4}$

The presentation of Kikuchi-Fujimoto disease closely mimics that of lymphoma and is frequently misdiagnosed as such. Patients often undergo extensive investigations for infectious, neoplastic and autoimmune conditions before a diagnosis of Kikuchi-Fujimoto disease is considered. The differential diagnosis for this disease is shown in Box 2., Diagnosis cannot be made using standard laboratory or diagnostic modalities; a lymph node biopsy is required. Histologic criteria for Kikuchi-Fujimoto disease are listed in Box 3., 1,3,5

Kikuchi-Fujimoto disease has been found in association with various viruses, bacteria and parasites, but as of yet no

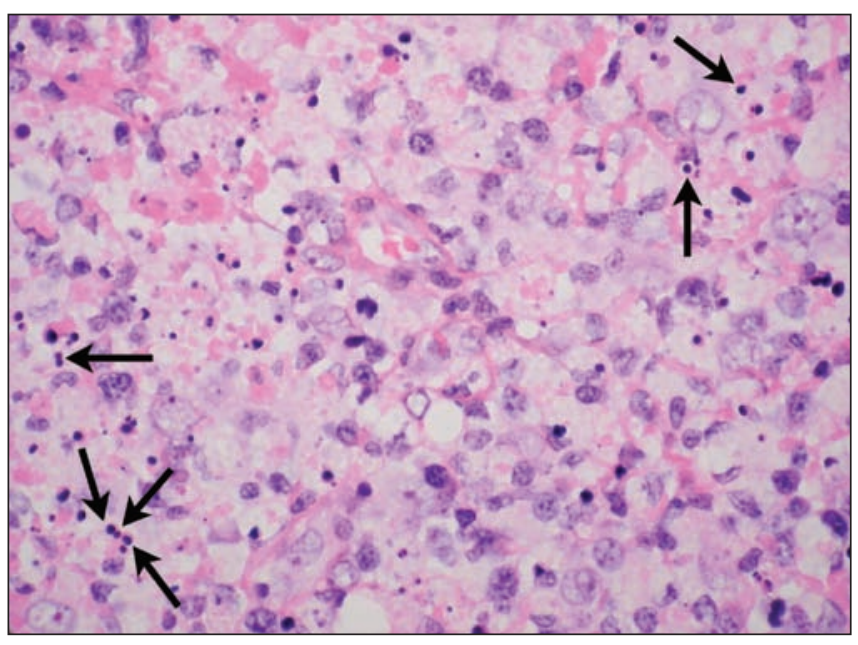

Figure 1: Microscopic view of lymph node biopsy specimen from Patient 2 (hematoxylin and eosin stain, magnification 630), showing reactive histiocytes containing darkly stained, spherical karyorrhectic debris (arrows), a lack of neutrophils or malignant cells, and relatively few plasma cells.

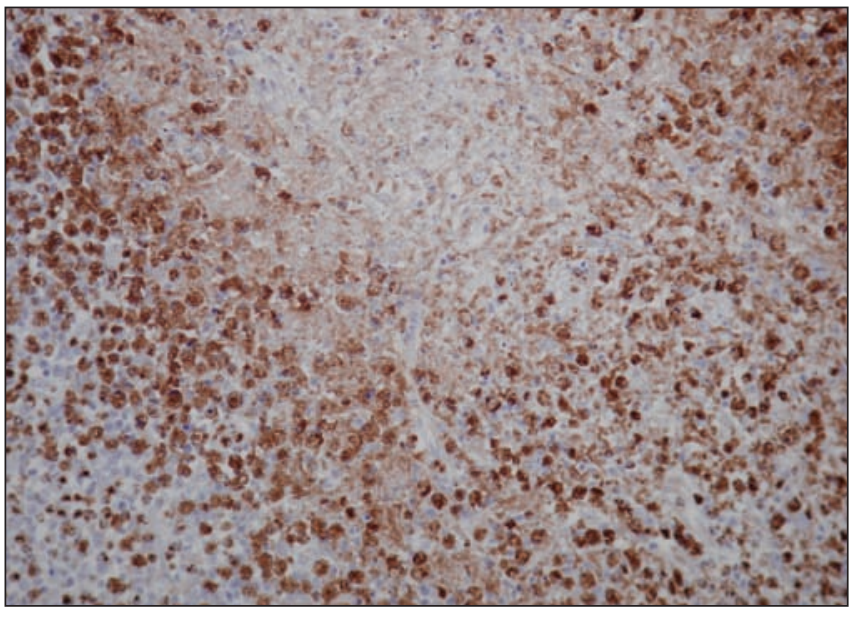

Figure 2: Microscopic view of lymph node biopsy specimen from Patient 2 (CD68 stain, magnification - 200), emphasizing the numerous histiocytes or macrophages with brown staining. 


\section{Box 1: Prevalence of the characteristics of Kikuchi-Fujimoto disease ${ }^{1}$}

- Lymphadenopathy (100\%)

- Leukopenia (43\%)

- Elevated erythrocyte sedimentation rate (40\%)

- Fever $(35 \%)$

- Anemia (23\%)

- Rash (10\%)

- Weight loss (10\%)

- Fatigue $(7 \%)$

- Joint pain (7\%)

- Night sweats, nausea, vomiting or diarrhea (<7\%)

clear causal relation has been shown. ${ }^{13,5}$ Kikuchi-Fujimoto disease shares many characteristics with systemic lupus erythematosus, including similar clinical, epidemiologic and histologic patterns. Patients who have had an episode of Kikuchi-Fujimoto disease may be at increased risk for systemic lupus erythematosus in the future, although the extent of this risk is unknown. ${ }^{6}$

\section{Human leukocyte antigen genotyping}

There are few reported instances of familial KikuchiFujimoto disease or familial associations in the published literature. ${ }^{4,7-9}$ Human leukocyte antigen (HLA) genotyping for familial Kikuchi-Fujimoto disease has been reported in only one paper. ${ }^{4}$ The sisters described in that case share some HLA genotypes as the sisters described here; both of our patients tested positive for HLA-Cw7 and the older sister (Patient 1) also tested positive for HLA-B35. Neither of these genotypes is known to be associated with an increased risk of nonfamilial Kikuchi-Fujimoto disease.?

Shared environmental exposure may also explain the coincident diagnosis of Kikuchi-Fujimoto disease in these sisters. Both siblings were from a remote Northern Ontario reserve and were Aboriginal. Given that they both had very poor dentition with associated periodontitis, perioral microorganisms may have triggered the development of this disease. However, no association between oral infection and development of Kikuchi-Fujimoto disease has been reported, so this hypothesis remains to be investigated.

\section{Conclusion}

Although uncommon, Kikuchi-Fujimoto disease should be considered in the differential diagnosis of cervical lymphadenopathy. In particular, it can be mistaken for malignant lymphoma. The contribution of genetic or environmental factors to the development of Kikuchi-Fujimoto disease remains unknown. Although the disease is self-limiting, long-term follow-up is important to monitor for recurrence of the disease or development of autoimmune disorders, such as systemic lupus erythematosis.

This article has been peer reviewed.

Competing interests: None declared.

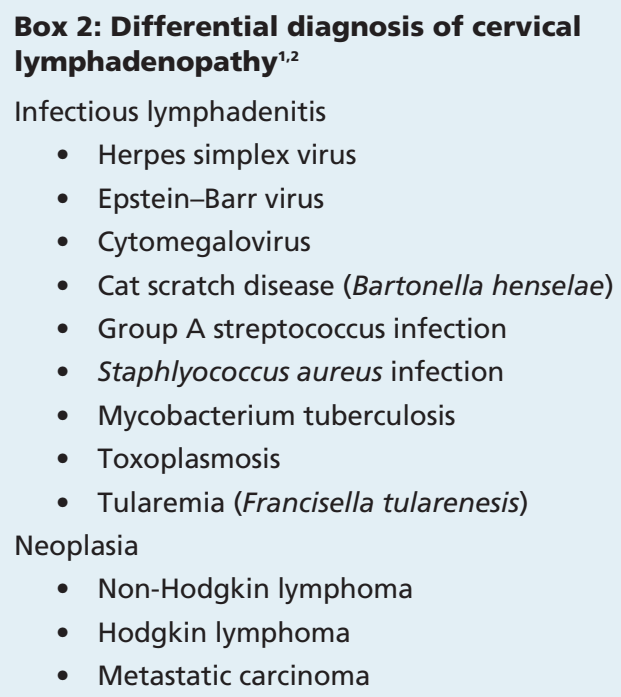

\section{Box 3: Features of Kikuchi-Fujimoto disease on lymph} node biopsy ${ }^{1,3,5}$

- Erosion of lymphatic tissue

- Circumscribed eosinophilic fibrotic necrotic foci with increased karyorrhexis

- Histiocytes surrounding the necrotic foci

- Nuclear debris

- Increased lymphocytes (predominantly CD8-positive T cells)

- Absence of granulocytes

- Paucity of plasma cells

\section{REFERENCES}

1. Kucukardali Y, Solmazgul E, Kunter E, et al. Kikuchi-Fujimoto disease: analysis of 244 cases. Clin Rheumatol 2007;26:50-4.

2. Dorfman RF, Berry GJ. Kikuchi's histiocytic necrotizing lymphadenitis: an analysis of 108 cases with emphasis on differential diagnosis. Semin Diagn Pathol 1988;5:329-45.

3. Lin HC, Su CY, Huang CC, et al. Kikuchi's disease: a review and analysis of 61 cases. Otolaryngol Head Neck Surg 2003;128:650-3.

4. Amir AR, Amr SS, Sheikh SS. Kikuchi-Fujimoto Disease: report of familial occurrence in two human-leukocyte antigen identical non-twin sisters. J Intern Med 2002;252:79-83.

5. Tsang WY, Chan JK, Ng CS. Kikuchi's lymphadenitis: a morphological analysis of 75 cases with special reference to unusual features. Am J Surg Pathol 1994;18: 219-31.

6. El-Ramahi KM, Karrar A, Ali MA. Kikuchi disease and its associations with systemic lupus erythematosus. Lupus 1994;3:409-11.

7. Ifeacho S, Aung T, Akinsola M. Kikuchi-Fujimoto disease: a case report and review of the literature. Cases $J$ 2008;1:187.

8. Atarashi K, Yoshimura N, Nodera H, et al. Recurrent histiocytic necrotizing lymphadenitis (Kikuchi's disease) in an human T lymphotropic virus type I carrier. Intern Med 1996;35:821-5.

9. Kikuchi M, Takeshita M, Eimoto T, et al. Histiocytic necrotizing lymphadenitis: clinicopathologic, immunologic, and HLA-typing study. In: Hanaoka M, Kadin M, Mikata A, editors. Lymphoid malignancy. New York (NY): Field and Wood; 1990. p. 251-7.

Correspondence to: Dr. Matthew D. Seftel, Section of Hematology-Oncology, Department of Internal Medicine, University of Manitoba, 675 McDermott Ave., Winnipeg MB R3E0V9; matthew.seftel@cancercare.mb.ca 